\title{
Towards Effective Bus Lane Monitoring Using Camera Sensors
}

\author{
$\mathrm{Xu} \mathrm{Li}{ }^{1}$, Xuegang $\mathrm{Yu}^{2 *}$, Ke $\mathrm{He}^{3}$ \\ ${ }^{1}$ Department of Computer Science and Engineering, State University of New York, Buffalo, USA \\ ${ }^{2}$ College of Computer Science and Technology, Jilin University, Jilin, China \\ ${ }^{3}$ Institute of Information Photonics and Optical Communications, Beijing University of Posts and \\ Telecommunications, Beijing, China \\ E-mail:frieyu@gmail.com \\ Received February 11, 2009; revised March 23, 2009; accepted April 1, 2009
}

\begin{abstract}
City administrators need to guarantee bus priority in urban public transportation. Building large-scale dedicated bus lanes is a cost-effective solution but it suffers from illegal utilization of dedicated bus lines by other non-permitted vehicles. In general, two systems can be utilized for bus lane monitoring: road-side system and bus mounted system. Although the former one has the advantage in terms of larger surveillance coverage, the investment cost makes it less feasible because of scalability issue. In this paper, we focus on bus mounted system to improve surveillance coverage without additional infrastructure cost. We introduce DoubleChecking, a cooperative violator identification scheme that can accurately pick out those non-permitted vehicles or violators. DoubleChecking is designed to improve the surveillance coverage of bus mounted system by using communications/cooperation between mounted camera sensors and existing camera sensors around intersections. Through theoretical analysis and simulation results, we show that DoubleChecking yields good performance for violator identification.
\end{abstract}

Keywords: Cooperative Violator Identification, Bus Lane Enforcement System, Sensors, ITS

\section{Introduction}

The rapid growth of modern society leads to an increasing demand for advanced public transit system. Bus Rapid Transit (BRT) is proposed to support bus-priority transportation, but it needs considerable infrastructure costs [1]. Another low-cost approach is to build dedicated bus lanes in urban area. However, the illegal utilizations by non-permitted vehicles (violators) degrade the effectiveness of dedicated bus lanes [2]. Fortunately, intelligent transportation systems (ITS) aim to use various modern technologies to improve road safety and urban traffic management [3-6].

In particular, bus lane enforcement systems have been introduced to identify the violators. A typical approach for violator identification is to use cameras and a key component in this system is the Number Plate Reader (NPR), by which the registration number of vehicle can be analyzed based on image processing. In general, there are two approaches to deploy the camera sensors: road-side mounted system and bus mounted system. In road-side mounted system, cameras are equipped in lampposts along the road, which could capture violators passing through bus lanes. Bus mounted system utilizes the cameras mounted on buses to identify and record these violators. The disadvantage of road-side mounted system is high infrastructure cost while the drawback of bus mounted system is the limited surveillance coverage.

In this paper, we are concerned with bus mounted system and interested in whether the surveillance coverage of bus mounted system can be increased without additional infrastructure cost. We propose DoubleChecking, a cooperative scheme for violator identification in bus lane enforcement system. Our solution utilizes the existing cameras around intersections and bus mounted cameras to enhance the probability of identifying the real violators. Specifically, a cooperative violator identification scheme with probabilistic guarantee is adopted based on wireless communications between cameras. Thus, we can identify not only the violator immediately in front of the bus (The number plate can be read directly, e.g., vehicle $A$ in Figure 1), but also the violator not 


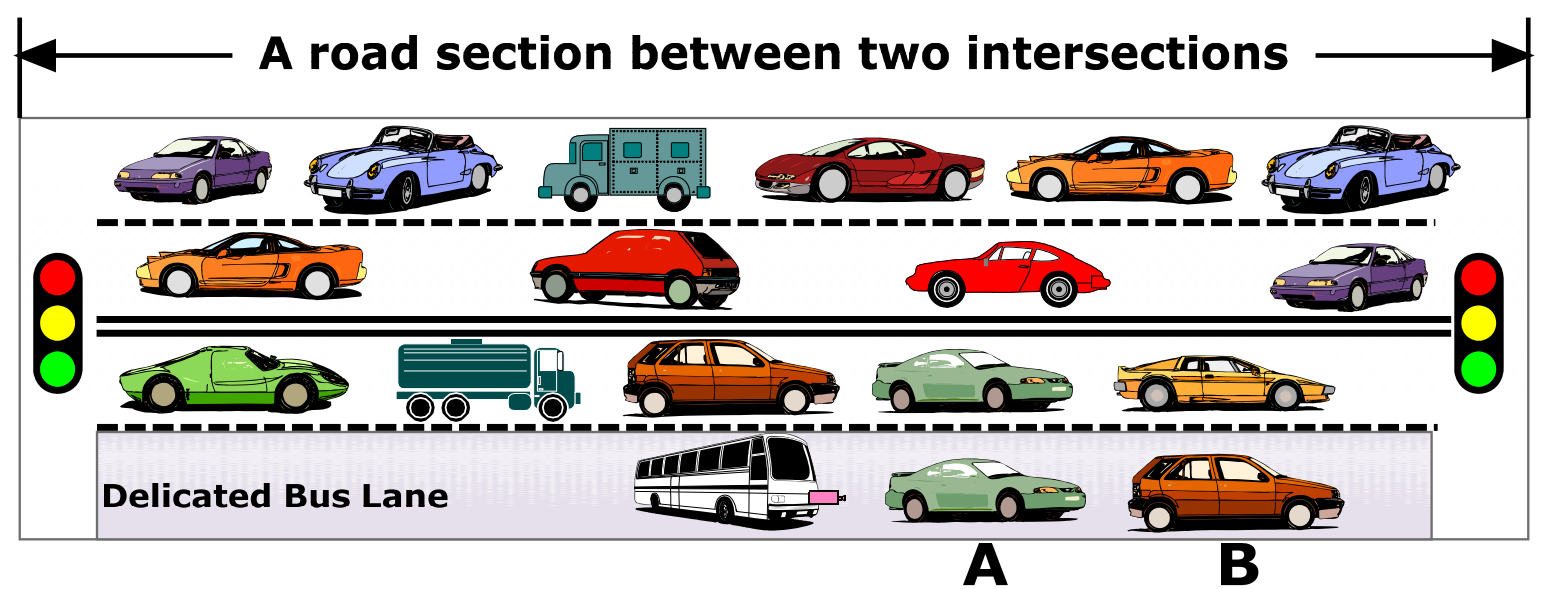

Figure 1. Application scenario of bus lane enforcement.

close to the bus (The buses cannot read the violator's number by bus mounted cameras directly because of sight blocking, e.g., vehicle $B$ in Figure 1). DoubleChecking essentially extends the surveillance coverage of bus mounted system without additional infrastructure cost. Overall, we mainly focus on novel architecture of bus lane enforcement system and the detailed image-processing issue is beyond the scope of this paper.

The remainder of the paper is organized as follows. Section 2 surveys the related work. Section 3 describes the assumptions, model and problem statement. In Section 4, we propose a novel DoubleChecking scheme for cooperative violator identification. Section 5 is the performance evaluation part, followed by the conclusion in Section 6.

\section{Related Work}

Recently, a new infrastructure named Vehicular Sensor Network (VSN) has attracted tremendous interests from both government and academic. VSN is a network of mobile sensors equipped on vehicles, such as taxis and buses [3,7-9], which can be used for urban sensing, such as traffic monitoring[10,11]. VSNs facilitate collection of surveillance data over a wider area than the fixed infrastructure $[6,12]$. Meanwhile, unlike traditional wireless sensor networks, vehicular sensors are typically not affected by strict energy constraints and vehicles can be equipped with powerful processing units and wireless transmitters. Actually, the work presented in this paper still falls into vehicular sensor networks do- main and the bus mounted cameras can be regarded as sensors.

Four bus lane enforcement projects have been carried out in United Kingdom: one in Birmingham and the other three in London $[2,13,14]$. The Birmingham Bus Lane Enforcement System depends on video camera and image processing equipment mounted on buses or along the roadside. The camera digitally takes photos for the vehicles in the bus lane twenty meters ahead of it and transmits the numbers of those vehicles to an E-display screen at the end of the bus lane. With this system, the bus lane offences decreased by $60 \%$ and average bus journey times decreased by $32 \%$ in Birmingham $[14,15]$. The Bus Lane Violation Detection \& Deterrent (BLVDD) in Heathrow is very similar to the roadside mounted system in Birmingham, which is installed on a highway to the airport [16]. The other two systems in London area rely on SVHS video cassette recorder rather than digital images to generate redundant information, with which more accurate violator identification can be expected [13]. Work in [17,18] studied the bus lanes enforcement with intermittent priority, in which the author proposed a cost effective approach to increase bus transit system speed and reliability without creating excessive delays to private vehicles.

\section{Scenario, Model and Problem Statement}

\subsection{Scenario and Road Section Model}

Actually, lots of buses are already equipped with various electronic devices by bus companies, such as GPS, storage device, wireless transmitter, etc. Besides that, we assume that two forward facing cameras (color and monochrome) and image processing unit are available on each bus. With a GPS system, a bus can determine whether or not it is in a bus lane and therefore whether or not to open the two bus mounted cameras [2]. At the same time, the existing static surveillance system around the intersections (including similar devices mentioned above) can be utilized to assist the bus mounted system for violator identification without additional infrastructure cost. Especially, a color camera provides a wide context view in front of the bus and a monochrome cam- 
era shows a close up view of the vehicle's number plate. An infra-red illuminator enables operation in poor lighting conditions. With an image processing unit, the registration number of vehicle can be analyzed. Due to sight blocking of bus mounted monochrome camera, it just could read the number of violator immediately in front of the bus. For the violators not close to the bus, only limited attribute information can be obtained by the wide context view of the bus mounted color camera, such as vehicle type, color, etc. However, the existing static cameras around intersections are capable of reading all the information of vehicles because of good viewpoint (high position, good orientation, as shown in Figure 1). In addition, with wireless communications, information can be exchanged between intersections or between buses and intersections to support cooperative violator identification.

In this work, we use a typical road section: a road section has three lanes and one of them is the dedicated bus lane (as illustrated in Figure 1). Meanwhile, we assume heavy traffic on the two public lanes and light traffic on the bus lane (It is not worth and necessary for public vehicle to be a violator if all lanes have similar traffic).

\subsection{Problem Statement}

In this work, we discuss the violator identification problem based on one-one model, i.e., a standard case unit includes one road section and one bus running on this road section. Accordingly, in each run of simulation, we only focus on one standard case unit. Specifically, we consider the following problem: it is known that we have a bus mounted system and an existing intersection system as introduced in Section 3.1, for a standard case unit, how to sort out a suspicious vehicle (to be the violator) set $\mathbf{S}$ by cooperative violator identification?

First, we introduce some definitions as follows.

Definition 1 (Maximum photographic distance): the definition is mainly used for bus mounted cameras, which have a limited field of vision. That is, they only can generate high quality images for limited distance in front of the bus. This parameter is named as maximum photographic distance, denoted as $\boldsymbol{m p d}$. How to regulate the orientation of camera for capturing image and how to deal with the image processing are beyond the scope of this paper, related works can be found in [19-21].

Definition 2 (Average accuracy ratio, $A A R$ ): for a standard case unit, we get a suspicious vehicle set $\boldsymbol{S}$ by cooperative violator identification. The accuracy ratio $(A R)$ is defined as the proportion of real violator in the set $\boldsymbol{S}$. Thus, for $\boldsymbol{W}$ standard case units, the average accuracy ratio is:

$$
A A R=\frac{1}{W} \sum_{j=1}^{W} \frac{\left|S_{j}^{\prime}\right|}{\left|S_{j}\right|}
$$

where $\left|S_{j}\right|$ and $\left|S_{j}^{\prime}\right|$ are the sizes of suspicious vehicle set $\boldsymbol{S}$ and the real violator vehicle set $\boldsymbol{S}^{\prime}$ in the $j^{\text {th }}$ standard case unit, respectively. The metric indicates the effectiveness of bus lane enforcement system. In addition, we define another vehicle set $\boldsymbol{S}^{\prime \prime}=\boldsymbol{S}-\boldsymbol{S}^{\prime}$, which includes the vehicles which are in the suspicious vehicle set but are not the real violators.

\section{Cooperative Violator Identification for Bus Lane Enforcement}

\subsection{Methodology}

On one hand, some attribute information of vehicles which are in $\boldsymbol{m p d}$ distance of a bus, can be identified based on image processing. Typically, attribute information includes vehicle type, color, brand, length, taxi or private vehicle, etc. To be general, in the paper, we assume that vehicle type and color information can be obtained. Meanwhile, the number of vehicle immediately in front of the bus can be obtained by monochrome camera. In addition, the bus can estimate the relative locations of violators in the whole traffic flow by GPS coordinates. All related information can be transmitted from bus to the forward intersection's processing unit by wireless communication, such as DSRC.

On the other hand, a road section is connected with two intersections. With utilization of cameras at two intersections, for a given unidirectional traffic flow, we can construct two vehicle sequences based on their timestamps of entering/exiting the road section, respectively (Accordingly, two indexes will be assigned to each vehicle in both entering/exiting sequences and large index corresponds to large timestamp). Basically, the two indexes of a vehicle should not have considerable change in two sequences due to the heavy traffic on the public lanes, which is similar with FIFO. However, for an exceptional vehicle which is with large index in entering sequence while small index in exiting sequence (i.e., compared with other vehicles, it took less time to travel through the road section), it has higher probability to have illegally utilized the bus lane as a violator.

Finally, with cooperation between intersections and buses, a fraction of traffic flow can be selected based on the location information of violators provided by bus. Then, the exceptional vehicles in this targeted traffic flow will be examined by utilizing more information sent from the bus, such as vehicle type and color, etc. 


\subsection{DoubleChecking Scheme}

We first give the definition of exceptional vehicle.

Definition 3 (Exceptional vehicle): for a given threshold $\delta$, the vehicle $v$ will be regarded as an exceptional vehicle if and only if:

$$
\text { v.eni-v.exi } \geq \delta
$$

where $v$.eni and $v$.exi are the two indexes of $v$ in the entering and exiting sequences, respectively. Actually, here we are only interested in the exceptional vehicles which have less travelling time costs than others. The threshold $\delta$ is an empirical parameter based on traffic flow model, which indicates how sensitive for the DoubleChecking scheme to define exceptional vehicles.

Based on the discussion in Section 4.1, we propose DoubleChecking, a novel cooperative violator identification scheme for Bus Lane Enforcement, as shown in Figure 2. In addition, we admit there are still exceptional vehicles or violators which cannot be monitored by buses because of limited $\boldsymbol{m p d}$ of bus mounted cameras. Actually, with increase of bus density, we may enable the communication and cooperation between buses, which will further improve the surveillance coverage of bus lane enforcement system. Meanwhile, the model used in this paper can also be extended so that the bus may

Scheme DOUBLECHECKING:

// For a given bus $b$ and road section with length $l$ in standard case unit

For a bus $b$, the bus mounted cameras take photographs for the violators (denoted as $v_{i}$ ) in its maximum photographic distance. For each violator $v_{i}$, with utilization of GPS and speed estimator, the bus can calculate the time instant $T_{i}$, at which $v_{i}$ will arrive at the forward intersection (corresponding to parameter $T_{\text {begin }}$ at the intersection). Other information can be also analyzed by image processing, such as vehicle type, color. Especially, the number plate can be read if the violator is immediate in front of the bus (denoted as $v^{\prime}$ ). Then, All the related information, e.g. ( $v_{i}$, type, color $)$, will be transmitted to the forward intersection's processing unit by wireless communication.

For the forward intersection of bus $b$, currently there are two time-related parameters: $T_{\text {begin }}$ and $T_{\text {end }}$. If the intersection receives a new time instant $T_{j}$ from bus $b$ and $T_{j}$ is earlier than $T_{\text {begin }}$, then $T_{\text {begin }}$ will be updated by $T_{j} . T_{\text {end }}$ is the time instant, at which $v^{\prime}$ will arrive at the forward intersection.

The traffic flow between $\left[T_{\text {begin }}, T_{\text {end }}\right]$ will be examined for violator identification. The exceptional vehicles in this traffic flow will be sorted out based on Definition 3, which constitute a vehicle set, denoted as $\boldsymbol{S}^{\mathbf{0}}$. (First Checking Process)

For each vehicle in set $S^{0}$, it will be added into final suspicious vehicle set $\boldsymbol{S}$ if it has same (type, color) combination provided by bus $b$. (Second Checking Process)

Figure 2. The DoubleChecking scheme. monitor the violators behind it. Then, according scheme can be designed based on DoubleChecking. We state this part as our future work.

\section{Performance Evaluation}

\subsection{Theoretical Analysis}

In this section, we carried out a theoretical analysis about expectation of accuracy ratio. For a standard case unit, we have the following parameters:

$N$ : number of vehicles in the whole traffic flow;

$\alpha$ : the proportion of exceptional vehicle in the traffic flow (not including the real violator), this parameter is related to the parameter $\delta$;

$\boldsymbol{n}$ : number of real violators in suspicious vehicle set $\boldsymbol{S}$;

$t \boldsymbol{p}$ : number of vehicle types;

$\boldsymbol{c l r}$ : number of vehicle colors;

$\boldsymbol{\beta}$ : the proportion of traffic flow used for violator identification by DoubleChecking. Actually, this parameter is related to the parameters $T_{\text {begin }}$ and $T_{\text {end }}$ (Step 3 in Figure 2).

Accordingly, the number of exceptional vehicles in the targeted traffic flow is $N \times \alpha \times \beta$, which is denoted as $\boldsymbol{m}$. Now, we tend to calculate $E(|\boldsymbol{S}|)$ and $E(|A R|)$, first we have:

$$
\begin{gathered}
E(|\boldsymbol{S}|)=E\left(\left|\boldsymbol{S}^{\prime}\right|+\left|\boldsymbol{S}^{\prime \prime}\right|\right)=E\left(\left|\boldsymbol{S}^{\prime}\right|\right)+E\left(\left|\boldsymbol{S}^{\prime \prime}\right|\right)=n+E\left(\left|\boldsymbol{S}^{\prime \prime}\right|\right) \\
E\left(\left|\boldsymbol{S}^{\prime \prime}\right|\right)= \\
P\left(\left|\boldsymbol{S}^{\prime \prime}\right|=1\right) \times 1+P\left(\left|\boldsymbol{S}^{\prime \prime}\right|=2\right) \times 2+\cdots \\
+P\left(\left|\boldsymbol{S}^{\prime \prime}\right|=i\right) \times i \cdots+P\left(\left|\boldsymbol{S}^{\prime \prime}\right|=m\right) \times m
\end{gathered}
$$

Here, $\left|\boldsymbol{S}^{\prime \prime}\right|=i$ means there are $i$ out of $m$ exceptional vehicles in the suspicious vehicle set $\boldsymbol{S} . P\left(\left|\boldsymbol{S}^{\prime \prime}\right|=i\right)$ is the probability that the incident $\left|S^{\prime \prime}\right|=i$ happens. For given numbers of color $\boldsymbol{c l r}$ and vehicle type $\boldsymbol{t p}$, there are totally $\boldsymbol{t} \boldsymbol{p} \times \boldsymbol{c l r}$ combinations (In our work, we simply assume that each vehicle randomly chooses its type and color). Then, we have:

$$
\begin{aligned}
P\left(\left|\boldsymbol{S}^{\prime \prime}\right|=i\right)= & P\left(A_{1}\right) \times P\left(B_{1} \mid A_{1}\right)+\cdots \\
& +P\left(A_{j}\right) \times P\left(B_{j} \mid A_{j}\right)+\cdots \\
& +P\left(A_{i}\right) \times P\left(B_{i} \mid A_{i}\right)
\end{aligned}
$$

where incident $A_{j}$ means $i$ exceptional vehicles occupy $j$ color $\times$ type combinations and incident $B_{j}$ means that for each of $j$ color $\times$ type combinations in $A_{j}$, there exists real violator in the suspicious vehicle set $\boldsymbol{S}$, which has the same combinations.

Theorem 1: the probability that incident $A_{j}$ happens is:

$$
P\left(A_{j}\right)=\frac{C_{c l r \times t p}^{j} \times j^{i}-C_{c l r \times t p}^{j-1} \times(j-1)^{i}}{(c l r \times t p)^{i}}
$$


Here, the number of $j$-combinations (each of size $j$ ) from a set with $c l r \times t p$ elements (size $c l r \times t p$ ) is defined as:

$$
C_{c l r \times t p}^{j}=\frac{(c l r \times t p) !}{j ! \times(c l r \times t p-j) !}
$$

Proof: we use mathematical induction to proof our statement.

Basis: the Equation (2) holds for $j=1$.

$$
P\left(A_{1}\right)=\frac{C_{c l r \times t p}^{1}}{(c l r \times t p)^{i}}=\frac{C_{c l r \times t p}^{1} \times 1^{i}-C_{c l r \times t p}^{0} \times 0^{i}}{(c l r \times t p)^{i}}
$$

Inductive step: assume Equation (2) holds for $j \leq q$ :

$$
\begin{aligned}
P\left(A_{j}\right)= & \frac{C_{c l r \times t p}^{j} \times j^{i}-C_{c l r \times t p}^{j-1} \times(j-1)^{i}}{(c l r \times t p)^{i}}, j=1,2, \cdots, q \\
P\left(A_{q+1}\right)= & \frac{C_{c l r \times t p}^{q+1} \times(q+1)^{i}}{(c l r \times t p)^{i}}-\sum_{x=1}^{q} P\left(A_{x}\right) \\
= & \frac{C_{c l r \times t p}^{q+1} \times(q+1)^{i}}{(c l r \times t p)^{i}}-\sum_{x=1}^{q} \frac{C_{c l r \times t p}^{x} \times x^{i}-C_{c l r \times t p}^{x-1} \times(x-1)^{i}}{(c l r \times t p)^{i}} \\
= & \frac{C_{c l r \times t p}^{q+1} \times(q+1)^{i}}{(c l r \times t p)^{i}}-\left(\frac{C_{c l r \times t p}^{q} \times q^{i}}{(c l r \times t p)^{i}}-\frac{C_{c l r \times t p}^{q-1} \times(q-1)^{i}}{(c l r \times t p)^{i}}\right) \\
& -\left(\frac{C_{c l r \times t p}^{q-1} \times(q-1)^{i}}{(c l r \times t p)^{i}}-\frac{C_{c l r \times t p}^{q-2} \times(q-2)^{i}}{(c l r \times t p)^{i}}\right) \\
& -\cdots-\left(\frac{C_{c l r \times t p}^{2} \times 2^{i}}{(c l r \times t p)^{i}}-\frac{C_{c l r \times t p}^{1}}{(c l r \times t p)^{i}}\right)-\frac{C_{c l r \times t p}^{1}}{(c l r \times t p)^{i}} \\
= & \left(\frac{C_{c l r \times t p}^{q+1} \times(q+1)^{i}}{(c l r \times t p)^{i}}-\frac{C_{c l r \times t p}^{q} \times q^{i}}{(c l r \times t p)^{i}}\right) \\
& +\sum_{x=q-1}^{1} \frac{C_{c l r \times t p}^{x} \times x^{i}}{(c l r \times t p)^{i}}-\sum_{x=q-1}^{1} \frac{C_{c l r \times t p}^{x} \times x^{i}}{(c l r \times t p)^{i}} \\
= & \frac{C_{c l r \times t p}^{q+1} \times(q+1)^{i}}{(c l r \times t p)^{i}}-\frac{C_{c l r \times t p}^{q} \times q^{i}}{(c l r \times t p)^{i}}
\end{aligned}
$$

Since both the basis and the inductive step have been proved, it has now been proved by mathematical induction that Equation (2) holds for all $j$.

Theorem 2: the conditional probability of incident $B_{j}$ happens given $A_{j}$ is:

$$
P\left(B_{j} \mid A_{j}\right)=1-\sum_{x=1}^{j}(-1)^{x-1} C_{j}^{x}\left(\frac{c l r \times t p-x}{c l r \times t p}\right)^{i}
$$

Proof: we knew that incident $A_{j}$ means $i$ exceptional vehicles occupy $j$ color $\times$ type combinations, now we define incident $C_{l}$ means there is no real violator which occupies $l^{\text {th }}(l=1,2, \cdots, j)$ in $j$ color $\times$ type combinations occupied by $i$ exceptional vehicles. Thus,

$$
\text { Incident } \bigcup_{l=1}^{j} C_{l} \text { means there is at least one of } j
$$
color $\times$ type combinations, which is not occupied by a real violator. Then we have,

$$
\begin{gathered}
P\left(B_{j} \mid A_{j}\right)=1-P\left(\bigcup_{i=1}^{j} C_{l}\right) \\
P\left(\bigcup_{l=1}^{j} C_{l}\right)= \\
\sum_{l=1}^{j} P\left(C_{l}\right)-\sum_{1 \leq l<m \leq j} P\left(C_{l} C_{m}\right) \\
+\sum_{1 \leq l<m<n \leq j} P\left(C_{l} C_{m} C_{n}\right)+\cdots \\
+(-1)^{j-1} P\left(C_{1} C_{2} \cdots C_{j}\right)
\end{gathered}
$$

Actually, it is easy to calculate:

$$
P\left(C_{l}\right), P\left(C_{l} C_{m}\right), P\left(C_{l} C_{m} C_{n}\right) \cdots P\left(C_{1} C_{2} \ldots C_{j}\right)
$$

For example,

$$
\begin{gathered}
P\left(C_{1}\right)=\left(\frac{c l r \times t p-1}{c l r \times t p}\right)^{i} \\
P\left(C_{1} C_{2}\right)=\left(\frac{c l r \times t p-2}{c l r \times t p}\right)^{i} \\
P\left(C_{1} C_{2} \cdots C_{j}\right)=\left(\frac{c l r \times t p-j}{c l r \times t p}\right)^{i} \\
P\left(B_{j} \mid A_{j}\right)=1-P\left(\bigcup_{i=1}^{j} C_{i}\right)=1-\sum_{x=1}^{j}(-1)^{x-1} C_{j}^{x}\left(\frac{c l r \times t p-x}{c l r \times t p}\right)^{i}
\end{gathered}
$$

We proved the theorem 2 .

Then we have:

$$
\begin{aligned}
& E\left(\left|\boldsymbol{S}^{\prime \prime}\right|\right)=\sum_{i=1}^{m} P\left(\left|\boldsymbol{S}^{\prime \prime}\right|=i\right) \times i \\
& =\sum_{i=1}^{m}\left(\sum_{j=1}^{i} P\left(A_{j}\right) \times P\left(B_{j} \mid A_{j}\right)\right) \times i \\
& =\sum_{i=1}^{m}\left(\sum_{j=1}^{i}\left(\frac{C_{c l r \times t p}^{j} \times j^{i}-C_{c l r \times t p}^{j-1} \times(j-1)^{i}}{(c l r \times t p)^{i}}\right)\left(1-\sum_{x=1}^{j}(-1)^{x-1} C_{j}^{x}\left(\frac{c l r \times t p-x}{c l r \times t p}\right)^{i}\right)\right) \times i
\end{aligned}
$$




$$
\begin{aligned}
E(A R)= & \frac{E\left(\left|\boldsymbol{S}^{\prime}\right|\right)}{E(|\boldsymbol{S}|)}=\frac{n}{n+E\left(\left|\boldsymbol{S}^{\prime \prime}\right|\right)} \\
= & \left.\frac{n}{n+\sum_{i=1}^{m}\left(\sum_{j=1}^{i}\left(\frac{C_{c l r \times t p}^{j} \times j^{i}-C_{c l r \times t p}^{j-1} \times(j-1)^{i}}{(c l r \times t p)^{i}}\right)\right.} \times\left(1-\sum_{x=1}^{j}(-1)^{x-1} C_{j}^{x}\left(\frac{c l r \times t p-x}{c l r \times t p}\right)^{i}\right)\right) \times i
\end{aligned}
$$

We use a practical case unit as an example and the empirical parameter setting is as follows: there are totally $200(N)$ vehicles in this case, in which 5\% $(\boldsymbol{\alpha})$ are exceptional vehicles. Specially, $10 \%(\boldsymbol{\beta})$ of whole traffic flow is used for violator identification and the bus reports three $(\boldsymbol{n})$ vehicles as violators during travelling the road section in this case. The cameras can differentiate five (tp) vehicle types and five (clr) colors. Then, we calculated Equation (3) with values mentioned above and $E(A R)$ is about $97 \%$, which shows that high accuracy ratio can be expected by using DoubleChecking scheme.

\subsection{Simulation Verification}

We developed a traffic simulator using JAVA programming language. In each run of simulator, we focus on one standard case unit including one road section and a bus travelling on it, as shown in Figure 1. We generated the crowd traffic flow on the public lanes while the light traffic on the bus lane. Especially, the driving behaviors of bus and violators will be randomly chosen, such as speed, route, etc. Table 1 lists the default parameters used for the experiments in our simulation. The default values of experimental parameters are selected based on field experience. In addition, each data point in the following figures is averaged over 50 runs.

From Figure 3 to Figure 6, we plot the performance curves of DoubleChecking with different parameter settings. Overall, we can see that the DoubleChecking scheme has high average accuracy ratio (AAR) for violator identification. To be more precise, in the most of testing cases, nearly $90 \%$ vehicles in the suspicious vehicle set $\boldsymbol{S}$ are the real violators, which demonstrates the effectiveness of DoubleChecking.

Figures 3 and $\mathbf{4}$ show the AAR curve as offered road section length increases from $50 \mathrm{~m}$ to $500 \mathrm{~m}$ and maxi-

Table 1. The default values of experiment parameters.

\begin{tabular}{ll}
\hline Length of road section $(\boldsymbol{l})$ & $300 \mathrm{~m}$ \\
Max. Photographic dist. $(\boldsymbol{m p d})$ & $30 \mathrm{~m}$ \\
The proportion of exceptional vehicle $(\boldsymbol{\alpha})$ & $15 \%$ \\
No. of vehicle types $(\boldsymbol{t} \boldsymbol{p})$ & 5 \\
No. of vehicle colors $(\boldsymbol{c l r})$ & 5 \\
\hline
\end{tabular}

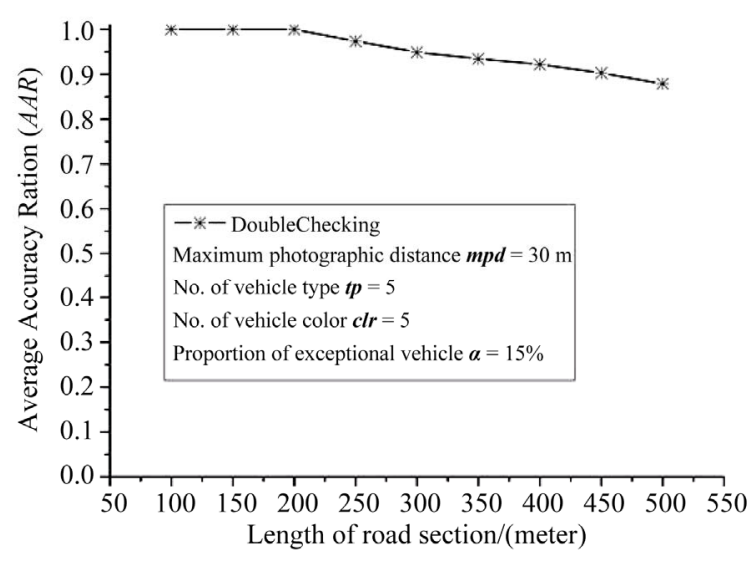

Figure 3. AAR with different lengths of road section.

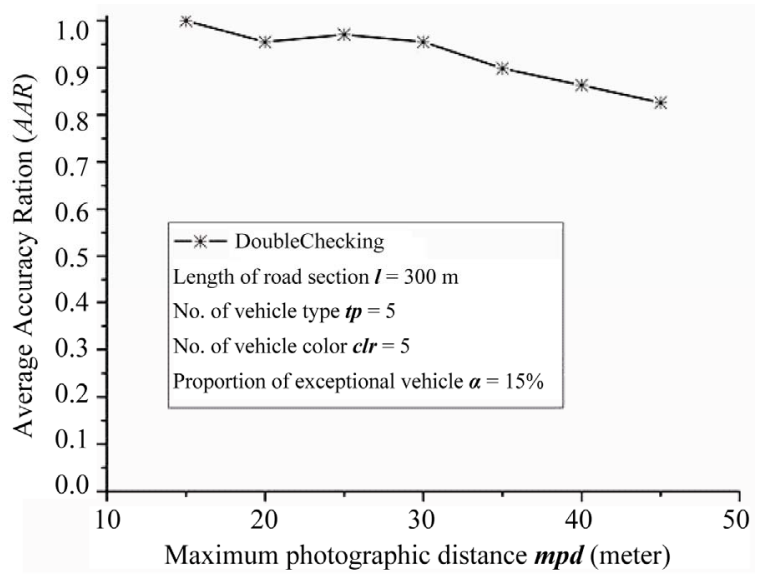

Figure 4. AAR with different $m p d$.

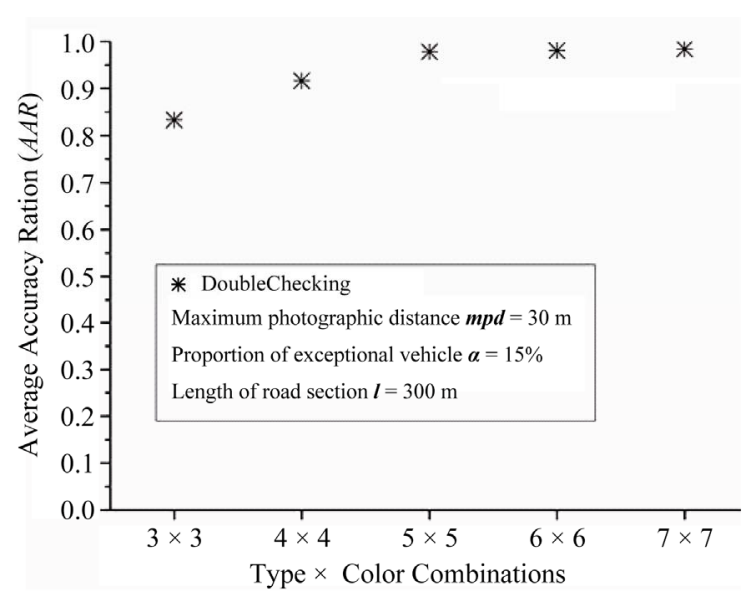

Figure 5. AAR with different type $\times$ color combinations.

mum photographic distance increases from $15 \mathrm{~m}$ to $45 \mathrm{~m}$, respectively. As shown in Figures 3 and 4, with increase of road length and maximum photographic distance, we can see a slow decrease in AAR. This phenomenon can be explained with the fact that with larger road length or 


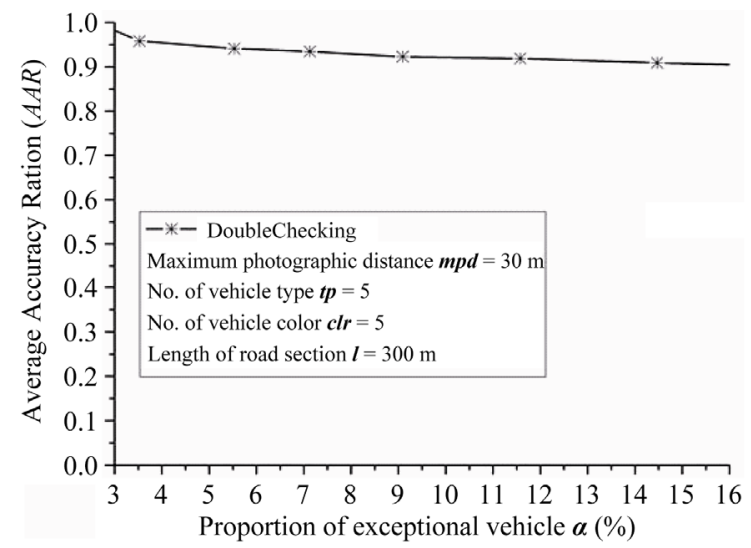

Figure 6. AAR with different proportions of exceptional vehicles.

maximum photographic distance, the bus will catch more violators during traveling, which leads to more type $\times$ color combinations involved. Finally, there is a higher probability for an exceptional vehicle to be included in the suspicious vehicle set $\boldsymbol{S}$. Conversely, Figure 5 shows the AAR curve as offered type $\times$ color increases from $3 \times$ 3 to $7 \times 7$. It is easy to understand that with increase of type and color numbers, there is an increase in AAR because large type $\times$ color combinations leads to a lower probability for an exceptional vehicle to have same (type, color) with any of the violators, due to the theoretical analysis in Section 5.1. In Figure 6, we can see that DoubleChecking has a good performance with different values of parameter $\alpha$. Specially, even if $20 \%$ vehicles in the whole traffic flow are exceptional vehicles $(\alpha=20 \%)$, high average accuracy ratio can still be expected, which shows the stability and robustness of our DoubleChecking scheme.

\subsection{Further Improvement}

As shown above, our DoubleChecking scheme already has high average accuracy ratio for violator identification. After that, we still can utilize a decision system as used in bus lane enforcement systems of UK $[15,16]$, to confirm the violators with more accuracy. In this system, we add the vehicles to a blacklist, which have been definitely identified as violators by DoubleChecking. It is easy to explain that a violator may often use the bus lane at any site. Thus, information sharing will be beneficial for global bus lane enforcement. If a vehicle in suspicious vehicle set $\boldsymbol{S}$ cannot be definitely identified as a violator by DoubleChecking but it matches a record in the blacklist, it will be regarded as a violator. Finally, the system will generate an information packet to be admissible as evidence in the courts for each violator, including the related images, time stamp, site description, etc.

\subsection{More Discussion about Bus Lane Enforcement and Practical Issues}

In this section, we tend to discuss some practical issues about bus lane enforcement in city urban area. Actually, the goal of dedicated bus lane is to improve the efficiency of city transportation system, especially for public transit. By deploying large-scale dedicated bus lanes in road network, we hope buses can travel with a higher speed. We point out that, however, there are also other factors which may decrease the mean speed of buses. Here, the most disadvantaged factor is traffic lights at intersections in city urban area. To validate the influence of traffic light, we carried out a real field testing, which is trip-based approach.

We design a route which crosses the urban area of Shanghai and take a taxi to finish our trip. The testing is carried out on May 29, 2007, we start from at 10:30 and arrive at end point at 13:09 with the whole trip of $56 \mathrm{~km}$ (Figure 7). The mean speed of whole trip is about 21.1 $\mathrm{km} / \mathrm{h}$. However, we calculate the sum of time cost due to traffic light delays at intersections, which almost come to 82 minutes whereas total time cost is 159 minutes. From the test, we see that nearly $51.2 \%$ of total time cost is waiting for red light, and the mean speed will be 43.8 $\mathrm{km} / \mathrm{h}$ if there is no traffic delay during the whole trip. This fact demonstrated that traffic light has a considerable influence on travelling speed of vehicles in city urban area. Accordingly, it will partially impair the advantages of dedicated bus lane system.

In addition, we admitted that the numerical investigations presented in this work are based on somewhat idealistic conditions and how the system will operate in a realistic setting still needs more effort. For instance, there is the case that some violator may turn right or left

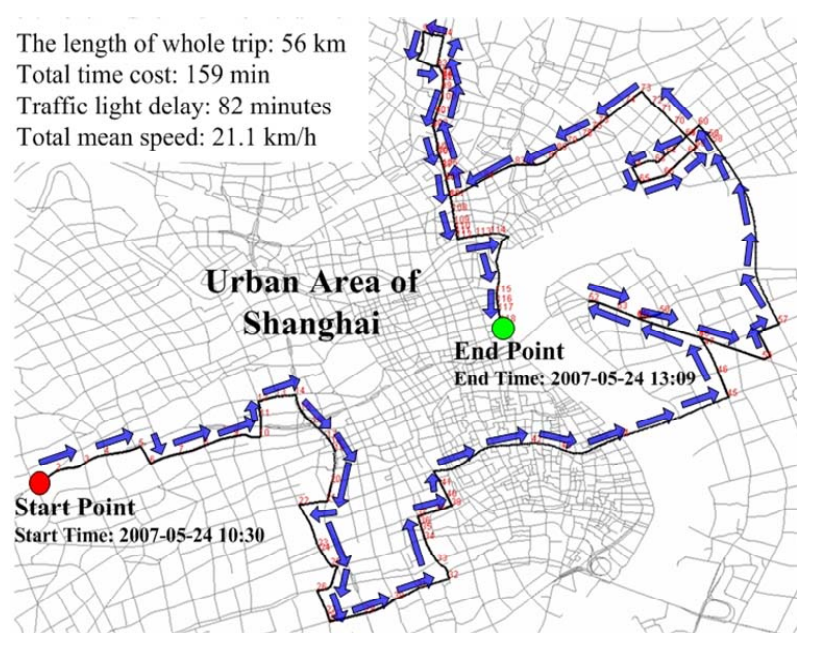

Figure 7. The influence of traffic light on mean travelling speed in urban area. 
and choose different routes so that they cannot be captured by the cameras at the fixed positions. The probability of this to happen could be capture in the mathematical analysis. We state it as a part of our future work.

Several issues remain to be addressed further. Our future work includes building a prototype system in Shanghai and testing our DoubleChecking scheme on this prototype. We hope the implementation experience helps us further to understand the efficiency of DoubleChecking. Second, DoubleChecking is a baseline scheme which can serve as guideline when deploying such an application in city urban area, how to design a more sophisticated violator identification scheme is also our future work (As discussed in Section 4.2, the model can be extended that the violators behind the bus can also be monitored. Meanwhile, we can enable the communication and cooperation between buses, which will further improve the surveillance coverage of bus lane enforcement system). These works are currently in progress in our lab.

\section{Conclusions}

We present a new scheme Doublechecking for bus lane enforcement system, which is designed to identify the violators in a cooperative manner. Compared with previous works, we tend to improve surveillance coverage of bus mounted system without additional infrastructure cost. To be more precise, we aim to identify not only the violator immediately in front of the bus, bus also the violators not close to the bus (The bus cannot read the violator's number directly because of sight blocking). With DoubleChecking scheme, the violators can be sorted out with high accuracy from traffic flow by the cooperation between bus mounted cameras and the existing cameras around intersections. From both theoretical performance analysis and simulation results, DoubleChecking shows a good performance for violator identification, which demonstrates the feasibility of our scheme.

\section{Acknowledgements}

This research was partially support by National Development and Reform Commission under Grant No. CNGI-09-01-11, and Jilin University under Grant No. 200903194.

\section{References}

[1] A. R. Girard, "Hybrid Supervisory Control for Real-Time Embedded Bus Rapid Transit Applications," IEEE Transactions on Vehicular Technology, Vol. 54, No. 5, 2005, pp. 1684-1696.
doi:10.1109/TVT.2005.853466

[2] T. Ellis, "Deterring Bus Lane Bandits," Traffic Technology International Annual Review, 1998, pp. 192-194.

[3] U. Lee, E. Magistretti, M. Gerla, P. Bellavista and A. Corradi, "Dissemination and Harvesting of Urban Data Using Vehicular Sensor Platforms," IEEE Transactions on Vehicular Technology, Vol. 58, No. 2, 2009, pp. 882-901. doi:10.1109/TVT.2008.928899

[4] M. Sede, X. Li, D. Li and M.-Y. Wu, M. L. Li and W. Shu, "Routing in Large-Scale Buses Ad Hoc Networks," IEEE Wireless Communications and Networking Conference, Las Vegas, March 31-April 3, 2008, pp. 2711-2716. doi:10.1109/WCNC.2008.475

[5] Y. Yang and R. Bagrodia, "Evaluation of VANET-based Advanced Intelligent Transportation Systems," Proceedings of the 6th ACM International Workshop on Vehicular Internet Working, New York, 2009, pp. 3-12.

doi: $10.1145 / 1614269.1614273$

[6] H. Z. Zhu, M. L. Li et al., "SEER: Metropolitan-Scale Traffic Perception Based on Lossy Sensory Data," IEEE INFOCOM 2009, Rio de Janeiro, 19-25 April 2009, pp. 217-225. doi:10.1109/INFCOM.2009.5061924

[7] B. Hull and V. Bychkovsky et al., "CarTel: A Distributed Mobile Sensor Computing System," Proceedings of the 4th International Conference on Embedded Networked Sensor Systems, Boulder, October 31-November 3, 2006. doi: $10.1145 / 1182807.1182821$

[8] J. Eriksson, H. Balakrishnan and S. Madden, "Cabernet: Vehicular Content Delivery Using WiFi," Proceedings of the 14th ACM International Conference on Mobile Computing and Networking, San Francisco, 14-19 September 2008, pp. 199-210. doi:10.1145/1409944.1409968

[9] A. Thiagarajan, L. Ravindranath et al., "VTrack: Accurate, Energy-Aware Road Traffic Delay Estimation Using Mobile Phones," Proceedings of the 7th ACM Conference on Embedded Networked Sensor Systems, Berkeley, November 2009. doi:10.1145/1644038.1644048

[10] X. Li, W. Shu et al., "Performance Evaluation of Vehicle-Based Mobile Sensor Networks for Traffic Monitoring," IEEE Transactions on Vehicular Technology, Vol. 58, No. 4, 2009, pp. 1647-1653. doi:10.1109/TVT.2008.2005775

[11] Xu Li et al., "Traffic Data Processing in Vehicular Sensor Networks," Proceedings of 17th International Conference on Computer Communications and Networks, St. Thomas, 3-7 August 2008, pp. 1-5. doi:10.1109/ICCCN.2008.ECP.42

[12] X. Li, H. Huang et al., "VStore: Towards Cooperative Storage in Vehicular Sensor Networks for Mobile Surveillance," IEEE Wireless Communications and Networking Conference, Budapest, 5-8 April 2009, pp. 1-6. doi:10.1109/WCNC.2009.4918021

[13] D. Turner and P. Monger, "The Bus Lane Enforcement Cameras Project: The London Area Scheme," Traffic Engineering \& Control, Vol. 38, No. 10, 1997, pp. 529-539.

[14] S. Lewis, "The Bus Lane Enforcement Cameras Handbook (Provisional)," Home Office, St Albans, 1996. 
[15] A. Wiggins, "Birmingham Bus Lane Enforcement System," 9th International Conference on Road Transport Information \& Control, 21-23 April 1998, pp. 80-84. doi:10.1049/cp:19980159

[16] G. Hill, "Bus Lane Violation Detection/Deterrent BLVDD," BAA Heathrow, 1998.

[17] M. D. Eichler, "Bus Lanes with Intermittent Priority: Assessment and Design," Masters of City Planning Thesis, Department of City and Regional Planning, University of California, Berkeley, 2005.

[18] M. D. Eichler, "Bus lanes with Intermittent Priority: Screening Formulae and an Evaluation," Working Paper UCB-ITS-VWP-2005-2, UC Berkeley Center for Future Urban Transport, 2005.
[19] S. Greenhill and S. Venkatesh, "Distributed Query Processing for Mobile Surveillance," Proceedings of the 15th International Conference on Multimedia, Augsburg, 24-29 September 2007, pp. 413-422. doi: $10.1145 / 1291233.1291331$

[20] B. Scheuermann, "A Fundamental Scalability Criterion for Data Aggregation in VANETs," Proceedings of the 15th Annual International Conference on Mobile Computing and Networking, Beijing, 20-25 September 2009, pp. 285-296. doi:10.1145/1614320.1614352

[21] S. Greenhill and S. Venkatesh, "Virtual Observers in a Mobile Surveillance System," Proceedings of the 14th Annual ACM International Conference on Multimedia, Santa Barbara, 23-27 October 2006, pp. 579-588. $\underline{\text { doi: } 10.1145 / 1180639.1180759}$ 